PHYSICAL REVIEW D 90, 026001 (2014)

\title{
Conformal symmetry for relativistic point particles
}

\author{
Roberto Casalbuoni* \\ Department of Physics and Astronomy, University of Florence and INFN, 50019 Florence, Italy \\ Joaquim Gomis ${ }^{\dagger}$ \\ Departament d'Estructura i Constituents de la Matèria and Institut de Ciències del Cosmos, \\ Universitat de Barcelona, Diagonal 647, 08028 Barcelona, Spain
}

(Received 29 April 2014; published 14 July 2014)

\begin{abstract}
In this paper we consider conformal dynamics for a system of $N$ interacting relativistic massless particles. A detailed study is done for the case of two particles, with a particular attention to the symmetries of the problem. In fact, we show that this analysis could be extended to the case of higher spin symmetries. Always in the two-particle case a formulation in terms of bilocal fields is proposed. For a system of $N$ particles we consider two possible scenarios: (i) the action is invariant under any permutation of the $N$ particles. This case corresponds to completely democratic interactions with each particle interacting with all the others. The action depends on $N-1$ dimensionless coupling constants. (ii) By putting the particles along a one-dimensional lattice (open or closed) with nearest neighbor interactions, one obtains a model with only two-body interactions depending on a single dimensionless coupling. This model can be easily extended to the continuum case, obtaining a conformal stringlike (closed or open) system.
\end{abstract}

DOI: 10.1103/PhysRevD.90.026001

PACS numbers: 11.25.Hf, 03.30.+p, 11.10.Ef, 11.30.-j

\section{INTRODUCTION}

The idea of conformal invariance in physics is more than one hundred years old (a very nice history of the conformal group can be found in H. A. Kastrup [1]). It started with $\mathrm{H}$. Bateman who in 1908 proved the invariance of the wave equation under inversion $x^{\mu} \rightarrow x^{\mu} / x^{2}$. This is a discrete transformation that, as we shall see, will play a crucial role in the present paper. A few months later, Biggs himself with two papers followed by one by E. Cunningham proved that the Maxwell equations are invariant under the conformal group. The next step was by $\mathrm{H}$. Weyl in 1918 , who tried to unify the gravitational and the electromagnetic interactions making use of conformal invariance. This approach was strongly criticized by Einstein. As it is well known this idea led eventually to the phase invariance of the Schrödinger equation and to the gauge invariance of the Maxwell theory.

The conformal invariance had a revival during the sixties and the seventies in two different areas, particle physics and critical phenomena. The interest in scale invariance in critical phenomena was raised from the works of L. P. Kadanoff [2] and Wilson and Kogut [3]. In particle physics the famous SLAC experiment on deep inelastic scattering aroused wide interest in scale symmetry and its extensions. The relevance of conformal symmetry in field theory was outlined by Polyakov [4] and used in the operator product expansion (OPE) by K. G. Wilson [5]. In the context of the OPE there was a revival of the idea of bootstrap (for a review see [6]).

\footnotetext{
casalbuoni@fi.infn.it † gomis@ecm.ub.es
}

Since then, the attention to conformal symmetry has always been very high. In field theory we recall the already mentioned invariance of the Maxwell equations and of the massless Dirac equation. At the classical level the scalar theory with a $\phi^{4}$ self-interaction and the non-Abelian YangMills theories are conformal invariant. In these cases the conformal invariance is broken by anomalies, but still it plays an important role. In condensed matter and in statistical field theory scale invariance at the critical points is a fundamental phenomenon. We also mention the importance of two-dimensional conformal symmetry in string theory and in general in two-dimensional field theories, where the conformal group, contrarily to the case of space dimensions different from two, is infinite dimensional. As a last point we mention the AdS/CFT correspondence [7-9] which allows us to define in a nonperturbative way $\mathrm{M} /$ string theory in terms of a (superconformal) quantum field theory in flat space-time. This idea has opened the possibility to study strongly coupled field theories in terms of gravitational theories.

It should be underlined that one of the main reasons that makes conformal theories so attractive is that they do not depend on any dimensionfull coupling constant.

More recently, conformal symmetry has become an important tool in the analysis of higher spin theories $[10,11]$; for a recent review see $[12,13]$. About this point it is interesting to notice that the higher spin symmetries of Vasiliev theory appear in the free massless Klein-Gordon equation [14]. At particle level these symmetries are all the symmetries of the action of a relativistic massless particle and they generalize the well-known conformal symmetries of this action. 
In this paper we present an application of conformal invariance to classical interacting relativistic particles. First of all, this problem, to the best of our knowledge, has been considered only in the nonrelativistic one-dimensional case. An example is the Calogero-Moser rational model [15-17], describing $N$ interacting particles via two-body interactions. This model is very important in the context of integrable models. For an extension to the supersymmetric case see, for example, [18]. The other example, always in one dimension, is in reference [19] where only one degree of freedom is considered but it contains a deep analysis of the role of the conformal group. For the superconformal case see [20].

The second point is that, after quantization, this theory is naturally connected with nonlocal field theories appearing in the context of higher spin theories; see for example [21]. As we mentioned previously, the underlying physics of the latter theories is connected with massless free particles. Since we are considering interacting massless particles, it would be worth trying to understand the possible connections.

This paper is organized as follows: after the introduction, in Sec. II we make use of the dilatation and translation invariance to show that a free massless particle cannot be described in configuration space proving that the Lagrangian vanishes identically. Therefore, in Sec. III we introduce Lagrange multipliers (or einbeins) in order to impose the mass zero condition. This is the description that we will use throughout this paper. Furthermore, using conformal invariance we write down an action for two relativistic massless particles in a $D$-dimensional space-time, with $D \neq 2$. In the case of two particles we further show that it is indeed possible to write down a Lagrangian using only the coordinate space. It turns out that this Lagrangian vanishes identically when turning off the coupling constant describing the interaction, as expected from the previous considerations. In this section we show also that there is a constraint in phase space involving the product of the momenta squared of the two particles.

Section IV is dedicated to the Hamiltonian analysis of the model. It turns out that there are two primary constraints and two secondary ones. We show that out of these four constraints two are first class and two second class. Eliminating the second class constraints through the use of the Dirac brackets we recover, in the reduced phase space, the constraint found previously in the Lagrangian analysis.

By construction, our model is explicitly invariant under the conformal group acting upon the coordinates of the two particles, but it is interesting to study in an explicit way the Killing vectors of the model. This is done in Sec. V, where we show that both the Killing vectors associated to the two particles satisfy the conformal Killing equations with independent infinitesimal parameters. However, due to the interaction, the two vectors must satisfy a further condition requiring that the infinitesimal parameters of the two
Killing vectors coincide. This implies an explicit breaking of the symmetry group of the free case $S O(D, 2)_{1} \otimes$ $S O(D, 2)_{2}$ to the diagonal subgroup $S O(D, 2)$.

The previous study is preliminary to what we do in Sec. VI, where we consider higher order Killing tensors. This means to take powers of the generator defined in the previous section. Obviously these powers are constant of motion, but there is some interest from the point of view of higher spin symmetries to study conformal Killing tensors [22]; see also the more recent papers [23,24]. In the case of free massless relativistic particles these symmetries are the enveloping algebra of the relativistic conformal group $[14,25]$. We study in particular the case of a Killing tensor of rank 2, deriving the conditions that must be satisfied to provide the required invariance. The equations we get are obviously satisfied when the Killing tensor is realized as the product of two Killing vectors, so the interest is to look for nonfactorized solutions. Is should also be noticed that in the case of higher spin the interest is in Killing vectors corresponding to a single space-time variable, whereas in our case they depend on two space-time variables (corresponding to the fact that we are studying a twoparticle system).

In Sec. VII we construct a bilocal field theory, involving two bilocal fields, such to incorporate the constraint found in Sec. II. This is also an interesting point since bilocal fields are naturally connected with higher spin symmetries.

In Sec. VIII we extend the two-particle model to $N$ massless particles interacting in a conformal invariant way. Here various possibilities open up according to the kind of symmetry we require under the exchange of the $N$ particles. In particular we will examine two models; in the first one we assume invariance with respect to any permutation among the $N$ particles. This entails a completely democratic model in which each particle interacts with all the others. The model depends on $N-1$ dimensionless coupling constants. We show that this model has the remarkable property of asymptotic separability. This should be understood in the following way: if we divide the $N$ particles in two clusters, one made up with $n$ and the other with $m$ particles, and we send to infinity all the distances among the particles of the first cluster and the particles of the second cluster, the original Lagrangian goes into the sum of two Lagrangians of the same kind as the original one. In the second model considered here, we associate the particle labels to the sites of a one-dimensional lattice, assuming nearest neighbor interactions. Therefore only two-body interactions are involved and the model is defined by a single dimensionless coupling. The asymptotic separability holds also in this case. There are not symmetries related to the exchange of particles. However, for a closed lattice there is a symmetry under discrete translations.

In Sec. IX we draw some conclusions and give an outlook for further problems to be studied. 


\section{CONFORMAL INVARIANCE IN PARTICLE COORDINATES}

We will discuss the requirements coming from conformal invariance on the Lagrangian of classical relativistic point particles. Let us start with one particle. We will prove that a conformal invariant Lagrangian for a single relativistic particle vanishes identically. Actually it will be enough to assume that the action is parametrization invariant and that it depends only on the coordinates of the particle. The generator of dilatations for a single particle is given by

$$
D=x_{\mu} p^{\mu}=-x_{\mu} \frac{\partial L}{\partial \dot{x}_{\mu}} \text {. }
$$

Notice the minus sign in the definition of the canonical momentum. This follows from our choice of a mostly minus metric $g_{\mu \nu}=(+,-,-, \cdots,-)$ in a $D$-dimensional space-time. We require $D$ in the previous equation to be a constant of motion and, furthermore, that the Lagrangian is homogeneous of first degree in the time parameter. It follows that

$$
0=\frac{d D}{d \tau}=-\dot{x}_{\mu} \frac{\partial L}{\partial \dot{x}_{\mu}}-x_{\mu} \frac{\partial L}{\partial x_{\mu}}=-L
$$

where we have used the Lagrange equations of motion

$$
\frac{d}{d \tau} \frac{\partial L}{\partial \dot{x}_{\mu}}=\frac{\partial L}{\partial x_{\mu}}=0
$$

and the invariance under translations. Therefore, the only solution for $D$ to be a constant in time is that the Lagrangian vanishes. It is obvious that this result applies to the case of $N$ noninteracting particles (under the same assumptions). This is an important point, since, if we want to consider a conformal invariant theory for a given number of particles, we cannot describe the free case using only space-time variables. In fact, as it is well known, a massless particle is described using an einbein variable defined on the world line (in practice a Lagrange multiplier). Therefore, this is the description that we will adopt, although for more than one particle a conformal invariant Lagrangian depending only on the coordinates can be constructed. On the other hand this latter formulation is such that, turning off the interaction, the Lagrangian vanishes identically, as it should be clear from the previous discussion.

\section{FORMULATION WITH THE EINBEINS}

The Lagrangian for a single free massless particle can be obtained through the use of an einbein $e$ :

$$
S=-\int d \tau \frac{\dot{x}^{2}}{2 e}
$$

from which varying with respect to the einbein we get the equation $\dot{x}^{2}=0$ and evaluating the momentum $p_{\mu}=\dot{x}_{\mu} / e$ we obtain $p^{2}=0$. The minus sign in front of the action is a consequence of our choice of the space-time metric. Requiring that the einbein transforms as a time derivative, this action is invariant under reparametrization. It is also invariant under Poincaré transformations. As for dilatations, we require

$$
x_{\mu} \rightarrow \lambda x_{\mu}, \quad e \rightarrow \lambda^{2} e .
$$

Furthermore, we recall that a special conformal transformation can be obtained through the following series of operations: (inversion) $\otimes($ translation $) \otimes($ inversion $)$; therefore, to impose the conformal symmetry it is enough to require the invariance under inversion,

$$
x^{\mu} \rightarrow \frac{x^{\mu}}{x^{2}}
$$

The transformation property of $\dot{x}^{2}$ is

$$
\dot{x}^{2} \rightarrow \frac{\dot{x}^{2}}{x^{4}},
$$

from which it follows

$$
e \rightarrow \frac{e}{x^{4}}
$$

Summarizing, the action (4) is invariant under conformal and reparametrization transformations.

Now let us discuss the case of two particles. We start at the free level with two massless particles

$$
S_{\text {free }}=-\int d \tau\left(\frac{\dot{x}_{1}^{2}}{2 e_{1}}+\frac{\dot{x}_{2}^{2}}{2 e_{2}}\right) \text {. }
$$

In order to construct an interaction term depending on the relative coordinate

$$
r_{\mu}=x_{1 \mu}-x_{2 \mu},
$$

we notice that under inversion

$$
r^{2} \rightarrow \frac{r^{2}}{x_{1}^{2} x_{2}^{2}}
$$

Therefore a conformal invariant action for two relativistic particles is given by

$$
S=-\int d \tau\left(\frac{\dot{x}_{1}^{2}}{2 e_{1}}+\frac{\dot{x}_{2}^{2}}{2 e_{2}}+\frac{\alpha^{2}}{4} \frac{\sqrt{e_{1} e_{2}}}{r^{2}}\right) .
$$

The variation with respect to the einbeins gives rise to the following equations: 


$$
\begin{gathered}
\frac{\partial L}{\partial e_{1}}=\frac{\dot{x}_{1}^{2}}{2 e_{1}^{2}}-\frac{\alpha^{2}}{8} \sqrt{\frac{e_{2}}{e_{1}}} \frac{1}{r^{2}}=0, \\
\frac{\partial L}{\partial e_{2}}=\frac{\dot{x}_{2}^{2}}{2 e_{2}^{2}}-\frac{\alpha^{2}}{8} \sqrt{\frac{e_{1}}{e_{2}}} \frac{1}{r^{2}}=0 .
\end{gathered}
$$

Resolving these two equations in the einbeins one finds

$$
\frac{1}{e_{1}}=\frac{\alpha}{2 \dot{x}_{1}^{2}}\left(\frac{\dot{x}_{1}^{2} \dot{x}_{2}^{2}}{r^{4}}\right)^{1 / 4}, \quad \frac{1}{e_{2}}=\frac{\alpha}{2 \dot{x}_{2}^{2}}\left(\frac{\dot{x}_{1}^{2} \dot{x}_{2}^{2}}{r^{4}}\right)^{1 / 4},
$$

with $\alpha \geq 0$. In extracting the square root we have chosen the minus sign, in order to have the time component of the canonical momenta with the same sign of the time derivative of the coordinate times, $x_{i}^{0}$. Substituting inside the action (12) we find

$$
S=-\alpha \int d \tau\left(\frac{\dot{x}_{1}^{2} \dot{x}_{2}^{2}}{r^{4}}\right)^{1 / 4} .
$$

As we have discussed previously the conformal invariant action for two particles in configuration space vanishes when the interaction is turned off.

Evaluating the momenta from (12) we get

$$
p_{i}^{\mu}=-\frac{\partial L}{\partial \dot{x}_{i \mu}}=\frac{\dot{x}_{i}^{\mu}}{e_{i}}
$$

The equations (13) can be expressed in terms of the momenta obtaining

$$
p_{1}^{2}-\frac{\alpha^{2}}{4} \sqrt{\frac{e_{2}}{e_{1}}} \frac{1}{r^{2}}=0, \quad p_{2}^{2}-\frac{\alpha^{2}}{4} \sqrt{\frac{e_{1}}{e_{2}}} \frac{1}{r^{2}}=0 .
$$

Finally, eliminating the ratio $e_{1} / e_{2}$ from these two equations we get a constraint among momenta and coordinates

$$
p_{1}^{2} p_{2}^{2}-\frac{\alpha^{4}}{16 r^{4}}=0 .
$$

This relation can also be obtained as a primary constraint from the action (15).

Notice that we have started with a flat metrics, $g_{\mu \nu}$, but we could have started with a conformal metrics as well, $g_{\mu \nu} \rightarrow \exp (2 \gamma(x)) g_{\mu \nu}$. In fact, in the formulation (12), the conformal factor can be absorbed into the definition of the einbeins, whereas the formulation (15) is explicitly scale invariant.

\section{HAMILTONIAN ANALYSIS}

In our notations the Poisson brackets among coordinates and momenta are

$$
\left\{x^{\mu}, p^{\nu}\right\}=-g^{\mu \nu}, \quad\left\{e_{i}, \pi_{j}\right\}=\delta_{i j} .
$$

Once again, the sign in the first Poisson bracket is fixed by our choice of the mostly minus metric. Notice also that there are two primary constraints

$$
\pi_{i}=\frac{\partial L}{\partial \dot{e}_{i}}=0 ;
$$

therefore the canonical Hamiltonian results to be

$H_{C}=-p_{1} \dot{x}_{1}-p_{2} \dot{x}_{2}-L=-\frac{e_{1}}{2} p_{1}^{2}-\frac{e_{2}}{2} p_{2}^{2}+\frac{\alpha^{2}}{4} \frac{\sqrt{e_{1} e_{2}}}{r^{2}}$.

Following Dirac we define the Dirac Hamiltonian, $H_{D}$, adding an arbitrary combination of the primary constraints $\pi_{i}=0$, in terms of two arbitrary functions $\lambda_{i}$,

$$
H_{D}=H_{C}+\lambda_{1} \pi_{1}+\lambda_{2} \pi_{2}
$$

Requiring the stability of the primary constraints we get two secondary constraints

$$
\begin{aligned}
& \left\{\pi_{1}, H_{D}\right\}=\frac{1}{2}\left(p_{1}^{2}-\frac{\alpha^{2}}{4} \sqrt{\frac{e_{2}}{e_{1}}} \frac{1}{r^{2}}\right) \equiv \phi_{1}, \\
& \left\{\pi_{2}, H_{D}\right\}=\frac{1}{2}\left(p_{2}^{2}-\frac{\alpha^{2}}{4} \sqrt{\frac{e_{1}}{e_{2}}} \frac{1}{r^{2}}\right) \equiv \phi_{2} .
\end{aligned}
$$

Notice that these two constraints are the same as the ones in (17). Then we have to consider the stability of the secondary constraints $\phi_{i}$, obtaining

$$
\begin{aligned}
\left\{\phi_{1}, H_{D}\right\}= & -\frac{\alpha^{2}}{4 r^{4}}\left(\sqrt{e_{1} e_{2}} p_{1} \cdot r+\sqrt{\frac{e_{2}^{3}}{e_{1}}} p_{2} \cdot r\right) \\
& +\frac{\alpha^{2}}{16 r^{2}}\left(\lambda \sqrt{\frac{e_{2}}{e_{1}^{3}}}-\lambda_{2} \frac{1}{\sqrt{e_{1} e_{2}}}\right), \\
\left\{\phi_{2}, H_{D}\right\}= & +\frac{\alpha^{2}}{4 r^{4}}\left(\sqrt{\frac{e_{1}^{3}}{e_{2}}} p_{1} \cdot r+\sqrt{e_{1} e_{2}} p_{2} \cdot r\right) \\
& +\frac{\alpha^{2}}{16 r^{2}}\left(\lambda_{2} \sqrt{\frac{e_{1}}{e_{2}^{3}}}-\lambda_{1} \frac{1}{\sqrt{e_{1} e_{2}}}\right) .
\end{aligned}
$$

These two constraints are not independent. In fact, the second equation can be obtained from the first one multiplying by $-e_{1} / e_{2}$. It follows that the stability of the secondary constraints can be attained by eliminating one of the two parameters $\lambda_{i}$, for instance, evaluating $\lambda_{1}$ from the first equation (24). We find

$$
\lambda_{1}=\frac{e_{1}}{e_{2}} \lambda_{2}+\frac{4}{r^{2}}\left(e_{1}^{2} p_{1} \cdot r+e_{1} e_{2} p_{2} \cdot r\right) .
$$

Correspondingly the Dirac Hamiltonian becomes 


$$
\begin{aligned}
H_{D}= & H_{C}+\frac{4}{r^{2}} e_{1}\left(e_{1} p_{1} \cdot r+e_{2} p_{2} \cdot r\right) \pi_{1} \\
& +\lambda_{2}\left(\pi_{2}+\frac{e_{1}}{e_{2}} \pi_{1}\right) .
\end{aligned}
$$

It is convenient to redefine $\lambda_{2}=\tilde{\lambda}_{2} e_{2}$, then

$$
H_{D}=H_{C}+\tilde{\lambda}_{2}\left(e_{1} \pi_{1}+e_{2} \pi_{2}\right)+C \pi_{1},
$$

with

$$
C=\frac{4}{r^{2}} e_{1}\left(e_{1} p_{1} \cdot r+e_{2} p_{2} \cdot r\right) .
$$

This expression suggests that the coefficient of $\lambda_{2}$ is a first class constraint. This can be verified by evaluating its Poisson bracket with $H_{D}$

$$
\left\{e_{1} \pi_{1}+e_{2} \pi_{2}, H_{D}\right\}=e_{1} \phi_{1}+e_{2} \phi_{2}-C \pi_{1} .
$$

This shows that the constraint $e_{1} \pi_{1}+e_{2} \pi_{2}$ is weakly stable. Then it is a simple algebra to prove that the two constraints

$$
e_{1} \pi_{1}+e_{2} \pi_{2}, \quad e_{1} \phi_{1}+e_{2} \pi_{2}-C \pi_{1}
$$

are weakly first class, that is their Poisson brackets with the other constraints $\pi_{1}, \pi_{2}, \phi_{1} \phi_{2}$ are proportional to one of these constraints. In conclusion, the four constraints can be divided as follows:

first class $e_{1} \pi_{1}+e_{2} \pi_{2}, \quad e_{1} \phi_{1}+e_{2} \phi_{2}-C \pi_{1}$,

second class $\pi_{1}, \quad \phi_{1}$.

Then, introducing the Dirac parentheses one can put $\pi_{1}$ and $\phi_{1}$ strongly to zero. In this way $\pi_{2}$ and $\phi_{2}$ turn out to be strongly first class.

The matrix of the second class constraints, $\chi_{i j}, i, j=$ 1,2 , is quite simple;

$$
\chi=\left(\begin{array}{cc}
0 & D \\
-D & 0
\end{array}\right), \quad \chi^{-1}=\left(\begin{array}{cc}
0 & -1 / D \\
1 / D & 0
\end{array}\right),
$$

where

$$
D=\left\{\phi_{1}, \pi_{1}\right\}=\frac{\alpha^{2}}{16} \sqrt{\frac{e_{2}}{e_{1}^{3}}} \frac{1}{r^{2}} .
$$

The Dirac brackets among any two dynamical variables are given by

$$
\begin{aligned}
\left\{O_{1}, O_{2}\right\}^{*}= & \left\{O_{1}, O_{2}\right\}+\frac{1}{D}\left[\left\{O_{1}, \phi_{1}\right\}\left\{\pi_{1}, O_{2}\right\}\right. \\
& \left.-\left\{O_{1}, \pi_{1}\right\}\left\{\phi_{1}, O_{2}\right\}\right] .
\end{aligned}
$$

In the reduced space using the second class constraints and the Dirac brackets the first class constraints become $\pi_{2}=0,\left.\quad \phi_{2}\right|_{\phi_{1}=0}=0$, implying $\left(p_{1}^{2} p_{2}^{2}-\frac{1}{16} \frac{\alpha^{4}}{r^{4}}\right)=0$,

and we recover Eq. (18) that was obtained previously by solving the equations for the einbeins.

Since among the two first class constraints we have one that is primary, it is known that we should have one gauge transformation. The generator of this gauge transformation can be constructed from a well-known algorithm; see for example [26-31]. The generator $G$, which is a constant of motion, is given by

$$
G=\sum_{i=1}^{2}\left(\frac{d}{d \tau}\left(\epsilon e_{i}\right) \pi_{i}-\left(\epsilon e_{i}\right) \phi_{i}\right),
$$

where $\epsilon(\tau)$ is an arbitrary function of the global parameter that parametrizes the two world lines.

The transformation generated by $G$ is

$$
\delta e_{i}=\frac{d}{d \tau}\left(\epsilon e_{i}\right), \quad \delta x_{i}^{\mu}=\epsilon \dot{x}_{i}^{\mu} ;
$$

it is the global world line diffeomorphism (Diff). Note that the interaction breaks the individual Diff invariance of the two world lines.

\section{ANALYSIS OF THE RIGID SYMMETRIES}

We have constructed our Lagrangian requiring conformal invariance, that is invariance under the group $S O(D, 2)$. Of course, when we consider two free massless particles, the invariance group is larger, namely it is the direct product of two conformal groups $S O(D, 2)_{1} \otimes$ $S O(D, 2)_{2}$ acting on the variables of the particles 1 and 2 respectively. When the interaction is introduced, the invariance is broken explicitly to the diagonal subgroup.

It is interesting to analyze these symmetries by looking at the conditions the Killing vectors must satisfy in order that our Lagrangian is invariant under the symmetries generated by generic Killing vectors

$$
G=\sum_{i=1}^{2} \xi_{i \mu}\left(x_{1}, x_{2}\right) p_{i}^{\mu} .
$$

In this section we will make use of the Lagrangian $L$, given in (15), in terms of which we have

$$
p_{i \mu}=-\frac{\partial L}{\partial \dot{x}_{i}^{\mu}}=-\frac{1}{2} \frac{\dot{x}_{i \mu}}{\dot{x}_{i}^{2}} L
$$

and the equations of motion

$$
\dot{p}_{1 \mu}=-\frac{\partial L}{\partial x_{1}^{\mu}}=+\frac{1}{2} \frac{r_{\mu}}{r^{2}} L, \quad \dot{p}_{2 \mu}=-\frac{\partial L}{\partial x_{2}^{\mu}}=-\frac{1}{2} \frac{r_{\mu}}{r^{2}} L .
$$


It is clear that the result will be that the Killing vectors are those of the conformal group, but the equations we will find here will be important for the analysis of the Killing tensors we will do in the next section. This analysis is relevant for the higher spin symmetries that have been recently considered in the literature [14,25].

By taking the time derivative of $G$, using the expression of the momenta given in Eq. (39) and the Lagrange equations of motion (40), we obtain

$$
\begin{aligned}
\dot{G}= & -\frac{1}{2} \sum_{i, j=1}^{2}\left(\partial_{j \mu} \xi_{i \nu}\left(x_{1}, x_{2}\right)\right) \frac{\dot{x}_{j}^{\mu} \dot{x}_{i}^{\nu}}{\dot{x}_{i}^{2}} L \\
& +\left(\xi_{1 \mu}\left(x_{1}, x_{2}\right)-\xi_{2 \mu}\left(x_{1}, x_{2}\right)\right) r^{\mu} \frac{L}{r^{2}}=0,
\end{aligned}
$$

where $\partial_{i \mu}=\partial / \partial x^{i \mu}$. Notice that the first term of this equation is not symmetric in $j$ and $i$.

A necessary condition to have a solution is

$$
\partial_{j \mu} \xi_{i \nu}\left(x_{1}, x_{2}\right)=0, \quad j \neq i,
$$

which implies that $\xi_{i \nu}=\xi_{i \nu}\left(x_{i}\right)$. If we use this information in $(41)$,

$$
\begin{aligned}
- & \frac{1}{2} \sum_{i=j}^{2}\left(\partial_{i \mu} \xi_{i \nu}\left(x_{i}\right)\right) \frac{\dot{x}_{i}^{\mu} \dot{x}_{i}^{\nu}}{\dot{x}_{i}^{2}} L \\
& +\left(\xi_{1 \mu}\left(x_{1}, x_{2}\right)-\xi_{2 \mu}\left(x_{1}, x_{2}\right)\right) r^{\mu} \frac{L}{r^{2}}=0 .
\end{aligned}
$$

Now the first term is symmetric in $\mu, \nu$. The solution of this equation is

$$
\begin{gathered}
\frac{1}{2}\left(\partial_{i \mu} \xi_{i \nu}\left(x_{i}\right)+\partial_{i \nu} \xi_{i \mu}\left(x_{i}\right)\right)=g_{\mu \nu} \lambda_{(i)}\left(x_{i}\right), \quad i=1,2, \\
\frac{1}{2} \sum_{i=1}^{2} \lambda_{(i)}=\left(\xi_{1 \mu}-\xi_{2 \mu}\right) r^{\mu} \frac{1}{r^{2}} .
\end{gathered}
$$

By contracting together the indices $\mu, \nu$ in (44) we find

$$
\lambda_{(i)}=\frac{1}{D} \partial_{i}^{\rho} \xi_{i \rho} .
$$

The two equations (44) tell us that $\xi_{1}^{\mu}$ and $\xi_{2}^{\mu}$ are the Killing vectors of two conformal groups $S O(D, 2)_{i}$ acting on the two variables $x_{1}$ and $x_{2}$ respectively. This is the symmetry group of two massless noninteracting particles. However, it is easily proved that the second condition (45) is satisfied if and only if the infinitesimal parameters defining the two Killing vectors are identical. Therefore the symmetry $S O(D, 2)_{1} \otimes S O(D, 2)_{2}$ is broken down to the diagonal subgroup $S O(D, 2)$ due to the interaction between the two particles.

\section{HIGHER SPIN SYMMETRIES}

In the previous section we have shown that the quantity $G$ [see (38)] is a constant of motion if the parameters defining the two conformal Killing vectors, corresponding to the two particles, are the same. It is a trivial observation that the power $G^{n}$ is also a constant of motion

$$
\frac{d G^{n}}{d \tau}=0 .
$$

The explicit expression for $G^{n}$ is

$$
G^{n}=\sum_{i_{1}, i_{2}, \cdots, i_{n}=1}^{2} \xi_{i_{1} \mu_{1}} \xi_{i_{2} \mu_{2}} \xi_{i_{n} \mu_{n}} p_{i_{1}}^{\mu_{1}} p_{i_{2}}^{\mu_{2}} \cdots p_{i_{n}}^{\mu_{n}}
$$

This defines a tensor of rank $n$ constructed in terms of the $n$ conformal Killing vectors in $n$ variables $x_{1}, x_{2}, \cdots, x_{n}$.

In principle, one could try to generalize the expression (48) to a generic Killing tensor [22]

$$
G^{\prime}=\sum_{i_{1}, i_{2}, \cdots, i_{n}=1}^{2} \xi_{i_{1} i_{2} \cdots i_{n}}^{\mu_{1} \mu_{2} \cdots \mu_{n}} p_{i_{1} \mu_{1}} p_{i_{2} \mu_{2}} \cdots p_{i_{n} \mu_{n}} .
$$

Notice that the tensor $\xi_{i_{1} i_{2} \cdots i_{n}}^{\mu_{1} \mu_{2} \cdots \mu_{n}}$ may depend on the variables $x_{1}, x_{2}, \cdots, x_{n}$. Requiring $G^{\prime}$ to be a conserved quantity one gets

$$
\begin{aligned}
0= & \sum_{k, i_{1}, i_{2}, \cdots, i_{n}=1}^{2} \partial_{k}^{\mu} \xi_{i_{1} i_{2} \cdots i_{n}}^{\mu_{1} \mu_{2} \cdots \mu_{n}} p_{i_{1} \mu_{1}} p_{i_{2} \mu_{2}} \cdots p_{i_{n} \mu_{n}} \dot{x}_{k \mu} \\
& +\sum_{j=1}^{n} \sum_{i_{1}, i_{2} \cdots, i_{n}=1}^{2} \xi_{i_{1} i_{2} \cdots i_{n}}^{\mu_{1} \mu_{2} \cdots \mu_{n}} p_{i_{1} \mu_{1}} p_{i_{j-1} \mu_{j-1}}\left((-1)^{i_{j}-1} \frac{r_{\mu}}{r^{2}} L\right) \\
& \times p_{i_{j+1} \mu_{j+1}} \cdots p_{i_{n} \mu_{n}},
\end{aligned}
$$

where we have used Eqs. (40) in the form

$$
\dot{p}_{i \mu}=(-1)^{i-1} \frac{r_{\mu}}{r^{2}} L
$$

Then, using (39)

$$
p_{i \mu}=-\frac{1}{2} \frac{\dot{x}_{i \mu}}{\dot{x}_{i}^{2}} L,
$$

$$
\begin{aligned}
0= & \frac{1}{2} \sum_{k, i_{1}, \cdots, i_{n}=1}^{2} \partial_{k}^{\mu} \xi_{i_{1} i_{2} \cdots i_{n}}^{\mu_{1} \mu_{2} \cdots \mu_{n}} \frac{\dot{x}_{i_{1} \mu_{1}} \dot{x}_{i_{2} \mu_{2}} \cdots \dot{x}_{i_{n} \mu_{n}} \dot{x}_{k \mu}}{\dot{x}_{i_{1}}^{2} x_{i_{2}}^{2} \cdots x_{i_{n}}^{2}} \\
& -\sum_{j=1}^{n} \sum_{i_{1}, i_{2} \cdots, i_{n}=1}^{2} \xi_{i_{1} i_{2} \cdots i_{n}}^{\mu_{1} \mu_{2} \cdots \mu_{n}} \frac{\dot{x}_{i_{1} \mu_{1}} \dot{x}_{i_{j-1} \mu_{j-1}}}{\dot{x}_{i_{1}}^{2} x_{i_{2}}^{2} \cdots x_{i_{j-1}}^{2}}\left((-1)^{i_{j}-1} \frac{r_{\mu}}{r^{2}}\right) \\
& \times \frac{\dot{x}_{i_{j+1} \mu_{j+1}} \cdots \dot{x}_{i_{n} \mu_{n}}}{\dot{x}_{i_{j+1}}^{2} \dot{x}_{i_{n}}^{2}} .
\end{aligned}
$$


Proceeding as in the previous section, one obtains equations for the tensor $\xi_{i_{1} i_{2} \cdots i_{n}}^{\mu_{1} \mu_{2} \cdots \mu_{n}}$ independent on $\dot{x}_{i}$. We know that these equations are satisfied when the tensor factorizes in $n$ conformal Killing vectors.

An interesting question remains open: is the factorized case the only solution to the previous equations?

Let us study in detail the case of $n=2$. We have

$$
\sum_{i j k=1}^{2}\left(\frac{1}{4} \partial_{k}^{\rho} \xi_{i j}^{\mu \nu} \frac{\dot{x}_{k \rho} \dot{x}_{i \mu} \dot{x}_{j \nu}}{\dot{x}_{i}^{2} \dot{x}_{j}^{2}}\right)-\sum_{i j=1}^{2}\left(\xi_{i j}^{\mu \nu}(-1)^{i-1} \frac{\dot{x}_{j \nu} r_{\mu}}{\dot{x}_{j}^{2} r^{2}}\right)=0 .
$$

Notice that this equation is not symmetric in $k, i, j$. Proceeding as in the previous section, the necessary condition to have a solution is

$$
\partial_{k}^{\rho} \xi_{i j}^{\mu \nu}=0, \quad k \neq i, \quad k \neq j
$$

using this condition we have

$$
\begin{aligned}
& \sum_{k=i, i \neq j}^{2} 2\left(\frac{1}{4} \partial_{i}^{\rho} \xi_{i j}^{\mu \nu} \frac{\dot{x}_{i \rho} \dot{x}_{i \mu} \dot{x}_{j \nu}}{\dot{x}_{i}^{2} \dot{x}_{j}^{2}}\right)+\sum_{k=i=j}^{2}\left(\frac{1}{4} \partial_{i}^{\rho} \xi_{i i}^{\mu \nu} \frac{\dot{x}_{i \rho} \dot{x}_{i \mu} \dot{x}_{i \nu}}{\dot{x}_{i}^{2} \dot{x}_{j}^{2}}\right) \\
& -\sum_{i \neq j}^{2}\left(\xi_{i j}^{\mu \nu}(-1)^{i+1} \frac{\dot{x}_{j \nu} r_{\mu}}{\dot{x}_{j}^{2} r^{2}}\right)-\sum_{i=j}^{2}\left(\xi_{i i}^{\mu \nu}(-1)^{i+1} \frac{\dot{x}_{i \nu} r_{\mu}}{\dot{x}_{i}^{2} r^{2}}\right)=0 .
\end{aligned}
$$

We get a solution requiring

$$
\begin{gathered}
\frac{1}{2}\left(\partial_{i}^{\rho} \xi_{i j}^{\mu \nu}+\partial_{i}^{\mu} \xi_{i j}^{\rho \nu}\right)=g^{\rho \mu} W_{(i j)}^{\nu}, \quad i \neq j \\
\partial_{i}^{\rho} \xi_{i i}^{\mu \nu}+\partial_{i}^{\mu} \xi_{i i}^{\rho \nu}+\partial_{i}^{\nu} \xi_{i i}^{\mu \rho}=g^{\rho \mu} V_{(i)}^{\nu}+g^{\mu \nu} V_{(i)}^{\rho}+g^{\nu \rho} V_{(i)}^{\mu} \\
\sum_{i=1}^{2}\left(2 \frac{1}{4} W_{(i j)}^{\nu}-\xi_{i j}^{\mu \nu}(-1)^{i+1} \frac{r_{\mu}}{r^{2}}\right)=0 \\
\sum_{i=1}^{2}\left(\frac{1}{4} V_{(i)}^{\nu}-\xi_{i i}^{\mu \nu}(-1)^{i+1} \frac{r_{\mu}}{r^{2}}\right)=0
\end{gathered}
$$

In these expressions

$$
W_{(i j)}^{\nu}=\frac{1}{2}\left(\partial_{i}^{\nu} \xi_{i j \mu}^{\mu}+\partial_{i \mu} \xi_{i j}^{\nu \mu}\right), \quad i \neq j,
$$

and

$$
V_{(i)}^{\nu}=\frac{1}{D+2}\left(\partial_{i}^{\nu} \xi_{i i \mu}^{\mu}+2 \partial_{i \mu} \xi_{i i}^{\mu \nu}\right) .
$$

In the factorized case

$$
\xi_{i j}^{\mu \nu}=\xi_{i}^{\mu}\left(x_{i}\right) \xi_{j}^{\nu}\left(x_{j}\right), \quad W_{i j}^{\nu}=\lambda_{(i)} \xi_{j}^{\nu}, \quad V_{i}^{\nu}=2 \lambda_{(i)} \xi_{i}^{\nu},
$$

where the $\lambda_{(i)} \mathrm{s}$ are defined in Eq. (46). It is easily verified that the previous equations (57), (58), (59) and (60) are satisfied. On the other hand, in principle it is possible that these equations have independent solutions, a fact that would be rather interesting.

\section{A BILOCAL FIELD THEORY}

Bilocal field theories have been considered recently in the framework of higher spin symmetries; see for example [21]. These bilocal field equations are conformal invariant; therefore it is of some interest to construct a bilocal conformal invariant field theory. This is made possible by encoding the constraint equation given in Eq. (18) in a bilocal field theory. To this end, let us introduce two bilocal fields, $\phi_{i}\left(x_{1}, x_{2}\right)$ with $i=1,2$. Then consider the action

$$
\begin{aligned}
S= & \int d^{4} x_{1} d^{4} x_{2}\left[\frac { 1 } { 2 } \left(\partial_{1 \mu} \phi_{1}\left(x_{1}, x_{2}\right) \partial_{1}^{\mu} \phi_{1}\left(x_{1}, x_{2}\right)\right.\right. \\
& \left.+\partial_{2 \mu} \phi_{2}\left(x_{1}, x_{2}\right) \partial_{2}^{\mu} \phi_{2}\left(x_{1}, x_{2}\right)\right) \\
& \left.-\phi_{1}\left(x_{1}, x_{2}\right) V\left(x_{1}, x_{2}\right) \phi_{2}\left(x_{1}, x_{2}\right)\right]
\end{aligned}
$$

where the potential $V$ is given by

$$
V\left(x_{1}-x_{2}\right)=\frac{\alpha^{2}}{4} \frac{1}{\left(x_{1}-x_{2}\right)^{2}} \text {. }
$$

Varying with respect to $\phi_{1}$ and $\phi_{2}$ we get the equations of motion

$$
\square_{1} \phi_{1}+V \phi_{2}=0, \quad \square_{2} \phi_{2}+V \phi_{1}=0 .
$$

Eliminating $\phi_{2}$ from the first equation

$$
\phi_{2}=-V^{-1} \square_{1} \phi_{1},
$$

and substituting inside the second one

$$
\square_{2}\left(V^{-1} \square_{1} \phi_{1}\right)-V \phi_{1}=0 .
$$

Then, multiplying by $V$, we obtain

$$
V \square_{2}\left(V^{-1} \square_{1} \phi_{1}\right)-V^{2} \phi_{1}=0 .
$$

Now let us look for solutions of the type

$$
\phi_{i}\left(x_{1}, x_{2}\right)=e^{i\left(p_{1} x_{1}+p_{2} x_{2}\right)} \tilde{\phi}_{i}\left(x_{1}-x_{2}\right) .
$$

Substituting inside the equations of motion (69) we find

$$
p_{1}^{2} \tilde{\phi}_{1}-V \tilde{\phi}_{2}=0, \quad p_{2}^{2} \tilde{\phi}_{2}-V \tilde{\phi}_{1}=0 .
$$

Eliminating again $\tilde{\phi}_{2}$ from the first one 


$$
\tilde{\phi}_{2}=V^{-1} p_{1}^{2} \tilde{\phi}_{1},
$$

and substituting inside the second one $\left(r=x_{1}-x_{2}\right)$

$$
\left(p_{1}^{2} p_{2}^{2}-\frac{\alpha^{4}}{16 r^{4}}\right) \tilde{\phi}_{1}\left(p_{1}, p_{2}, r\right)=0
$$

and an analogous equation for $\tilde{\phi}_{2}$. Notice that what we have done here is not to take the Fourier transform of the bilocal fields, but we have simply looked at a particular solution of the field equations.

As we have seen our theory gives rise to the constraint (18); therefore, in quantum theory it would be natural to transform it in a wave equation of the type

$$
\left(\square_{1} \square_{2}-\frac{1}{16} \frac{\alpha^{4}}{r^{4}}\right) \phi\left(x_{1}, x_{2}\right)=0,
$$

which should be looked at as a generalization of the conformal invariant massless Klein-Gordon equation to two conformal particles. On the other hand, this equation is fourth order in the derivatives and it might produce problems in a related field theory. In fact, higher order theories present, in general, ghosts in the spectrum. For this reason we prefer to start with a system of two fields, each of them obeying a second order equation.

An interesting point is to expand the equations of motion (69) in terms of a series of higher spin local fields, but we defer this problem to a future paper.

\section{CONFORMAL INVARIANT LAGRANGIANS FOR MANY PARTICLES}

In this section extend the case of a conformal invariant interaction between two particles to the case of $N$ particles. The kind of model one obtains depends on the symmetries one assumes in the exchange of the particles. We will start assuming the maximal symmetry, that is invariance under any permutation among the particles. This requirement and conformal invariance fix completely the interaction among the $N$ particles up to $N-1$ dimensionless couplings.

We start again from the einbein formulation for the Lagrangian describing $N$ massless free particles

$$
L_{\text {free }}=-\sum_{i=1}^{N} \frac{\dot{x}_{i}^{2}}{2 e_{i}}
$$

We recall from Sec. III that under inversion

$$
\dot{x}_{i}^{2} \rightarrow \frac{\dot{x}_{i}^{2}}{x_{i}^{4}}, \quad r_{i j}^{2} \rightarrow \frac{r_{i j}^{2}}{x_{i}^{2} x_{j}^{2}}, \quad i, j=1,2, \cdots N .
$$

In order for the free part to be invariant under inversion the einbeins must transform as

$$
e_{i} \rightarrow \frac{e_{i}}{x_{i}^{4}},
$$

whereas under reparametrization they must transform as a time derivative. In order to write down the invariant terms for the many particle case, let us notice that the two point interaction can be written in the following form:

$$
\left(\frac{e_{i} e_{j}}{r_{i j}^{2} r_{j i}^{2}}\right)^{1 / 2}
$$

This expression suggests that an invariant term for $n$ particles is of the form

$$
\left(\frac{e_{i_{1}} e_{i_{2}} \cdots e_{i_{n}}}{r_{i_{1} i_{2}}^{2} r_{i_{2} i_{3}}^{2} \cdots r_{i_{n-1} i_{n}}^{2} r_{i_{n} i_{1}}^{2}}\right)^{1 / n} .
$$

In fact, it is easily seen that this term is conformal invariant and transforms as a first derivative with respect to time.

Then the most general conformal invariant Lagrangian symmetric under the exchange of any pair of particles has the following structure:

$L=-\sum_{i=1}^{N} \frac{\dot{x}_{i}^{2}}{2 e_{i}}-\sum_{n=2}^{N} \beta_{n} \sum_{i_{1}<i_{2}<\cdots<i_{n}}\left(\frac{e_{i_{1}} e_{i_{2}} \cdots e_{i_{n}}}{r_{i_{1} i_{2}}^{2} r_{i_{2} i_{3}}^{2} \cdots r_{i_{n-1} i_{n}}^{2} r_{i_{n} i_{1}}^{2}}\right)^{1 / n}$,

$i_{k}=1,2, \cdots, N$.

It should be noticed that, differing with other theories involving $N$ particles, such as for instance the Calogero model [15], in our case we have not only two-body interactions but all the possible interactions among the $N$ particles; therefore, we have a real "democratic" model. It is rather interesting that this arises from the requirement of conformal symmetry.

If we imagine dividing the $N$ particles in two clusters of $\{n\}$ and $\{m\}$ particles, with $n+m=N$, the previous Lagrangian can be written in the form

$$
L_{N}=L_{n}+L_{m}+L_{n m},
$$

where $L_{n}$ and $L_{m}$ have exactly the same structure of $L_{N}$ and $L_{n m}$ contains all the terms involving distances between any particle of the set $\{m\}$ with any particle of the set $\{n\}$. Therefore, for very large distances among the particles belonging to the two different clusters, the term $L_{n m}$ goes to zero, that is

$$
\begin{aligned}
& \lim _{\text {for all } r_{i j}^{2} \rightarrow \infty} L_{N}=L_{n}+L_{m}, \\
& \text { such that } i \in\{n\}, \quad j \in\{m\} .
\end{aligned}
$$

This shows that this Lagrangian is separable for large distances among the two clusters. We could say that our Lagrangian satisfies the cluster decomposition at the classical level. 
In a complete analogous way we can get a conformal invariant Lagrangian without using the einbeins. The Lagrangian turns out to be

$L=-\sum_{n=2}^{N} \alpha_{i} \sum_{i_{1}<i_{2}<\cdots<i_{n}}\left(\frac{\dot{x}_{i_{1}}^{2} \dot{x}_{i_{2}}^{2} \cdots \dot{x}_{i_{n}}^{2}}{r_{i_{1} i_{2}}^{2} r_{i_{2} i_{3}}^{2} \cdots r_{i_{n-1}}^{2} i_{n} r_{i_{n} i_{1}}^{2}}\right)^{1 / 2 n}$.

Again this Lagrangian vanishes when all the interactions are turned off. We have not proved that this expression is obtained by eliminating the einbeins from the Lagrangian (80), but we conjecture that this is actually the case. The derivation of this result would be very useful in order to get the relation between the couplings $\beta_{i}$ and $\alpha_{i}$.

As a final observation we underline that the Lagrangian (80) depends on $N-1$ dimensionless couplings $\beta_{n}$ and that the number of terms involving $n$ particles out of $N$ is given by

$$
\left(\begin{array}{l}
N \\
n
\end{array}\right)
$$

Notice that the previous result applies also to the number of free terms (each of them involving one particle), by choosing $n=1$. Therefore, the total number of terms in (80) is given by

$$
\sum_{n=1}^{N}\left(\begin{array}{l}
N \\
n
\end{array}\right)=2^{N}-1
$$

Whereas in this model there are many particle interactions, one could consider a model with only two-body interactions. Imagine associating the particle labels with the sites of a one-dimensional lattice and consider only nearest neighbor interactions. The action would be

$$
S=-\int d \tau\left[\sum_{i=1}^{N} \frac{\dot{x}_{i}^{2}}{2 e_{i}}+\frac{\alpha^{2}}{4} \sum_{i=1}^{N-1} \frac{\sqrt{e_{i} e_{i+1}}}{r_{i, i+1}^{2}}\right]
$$

with

$$
r_{i, i+1}=x_{i}-x_{i+1} .
$$

Here we have assumed a single coupling. The asymptotic cluster decomposition holds also in this case. We could also consider a closed lattice identifying the first and the last particle. In this case the model has an obvious invariance under discrete translations. Furthermore, it can be extended to the continuum obtaining a conformal string (open or closed). This extension is actually under study and it will be the object of a different publication [32].

\section{CONCLUSIONS AND OUTLOOK}

In this paper we have studied what relativistic conformal symmetry can teach us about possible interactions among $N$ classical massless particles. The Lagrangian considered here depends on the symmetry we assume under the exchange of the particles. Assuming invariance under any permutation among the particles, the Lagrangian is completely fixed up to $N-1$ dimensionless coupling constants. This Lagrangian is rather interesting because it does not contain two-body interactions only, but, for any subset of $n$ particles out of the total set, it contains $n$-body interactions and it appears to be completely democratic. This is also shown by the number of terms in the Lagrangian, which does not grow with a power of $N$ but rather in an exponential way, namely like $2^{N}-1$. Another possibility that we have considered is the one corresponding to nearest neighbor interactions. The interest of this case is mainly related to the possibility of getting a simple limit in the continuum, obtaining in this way a conformal string [32].

We have analyzed the case $N=2$ with a particular emphasis on the symmetries. In fact, it is known that the conformal symmetry of the free massless Klein-Gordon equation can be extended to the enveloping algebra of the conformal group, obtaining in this way higher spin symmetries. In our case, we have two (or more) interacting particles preserving conformal symmetry, so a natural question to investigate is the possibility to enlarge the higher spin symmetries to interacting massless particles.

An interesting point is the extension of the conformal models presented here to superconformal ones [32].

The $N$ particle models could be considered in the case of $D=1$, that is a pure quantum mechanical case, in order to study their possible integrability. In particular, the nearest neighbor model looks similar to the Calogero-type models [15-17].

Another problem, to be investigated in the future, is the quantization of these models. A promising possibility, in our opinion, would be to try the world-line quantization, along the way paved by string theory. We recall here that the world-line quantization can be extended to the selfinteractions of scalar particles [33]. Another option is field quantization using, in the case of two particles, bilocal fields as introduced in Sec. VII.

\section{ACKNOWLEDGMENTS}

We thank Eric Berghsoeff and Jaume Gomis for their helpful comments. J. G. acknowledges the hospitality at the Department of Theoretical Physics of the University of Groningen, where this work was started. This work is partially financed by the Dutch research organization FOM and FPA Grants No. 2010-20807 and No. 2009 SGR502, and CPAN, Consolider Grant No. CSD 2007-0042. 
[1] H. A. Kastrup, Ann. Phys. (Amsterdam) 17, 631 (2008).

[2] L. P. Kadanoff, Physics (Long Island City, N.Y.) 2, 263 (1966).

[3] K. G. Wilson and J. B. Kogut, Phys. Rep. 12, 75 (1974).

[4] A. M. Polyakov, Pis'ma Zh. Eksp. Teor. Fiz. 12, 538 (1970) [JETP Lett. 12, 381 (1970)].

[5] K. G. Wilson, Phys. Rev. 179, 1499 (1969).

[6] S. Ferrara, R. Gatto, and A. F. Grillo, Springer Tracts Mod. Phys. 67, 1 (1973).

[7] J. M. Maldacena, Adv. Theor. Math. Phys. 2, 231 (1998).

[8] S. S. Gubser, I. R. Klebanov, and A. M. Polyakov, Phys. Lett. B 428, 105 (1998).

[9] E. Witten, Adv. Theor. Math. Phys. 2, 253 (1998).

[10] M. A. Vasiliev, Phys. Lett. B 243, 378 (1990).

[11] M. A. Vasiliev, Phys. Lett. B 567, 139 (2003).

[12] V. E. Didenko and E. D. Skvortsov, arXiv:1401.2975.

[13] M. A. Vasiliev, arXiv:1404.1948.

[14] M. G. Eastwood, Ann. Math. 161, 1645 (2005).

[15] F. Calogero, J. Math. Phys. (N.Y.) 12, 419 (1971).

[16] J. Moser, Adv. Math. 16, 197 (1975).

[17] M. A. Olshanetsky and A. M. Perelomov, Phys. Rep. 94, 313 (1983).

[18] D. Z. Freedman and P. F. Mende, Nucl. Phys. B344, 317 (1990).
[19] V. de Alfaro, S. Fubini, and G. Furlan, Nuovo Cimento A 34, 569 (1976).

[20] S. Fubini and E. Rabinovici, Nucl. Phys. B245, 17 (1984).

[21] D. Sorokin, AIP Conf. Proc. 767, 172 (2005).

[22] B. Carter, Phys. Rev. 174, 1559 (1968).

[23] M. Cariglia, G. W. Gibbons, J.-W. van Holten, P. A. Horvathy, P. Kosinski, and P.-M. Zhang, Classical Quantum Gravity 31, 125001 (2014).

[24] M. Cariglia, G. W. Gibbons, J.-W. van Holten, P. A. Horvathy, and P.-M. Zhang, arXiv:1404.3422.

[25] A. Y. Segal, Nucl. Phys. B664, 59 (2003).

[26] L. Castellani, Ann. Phys. (N.Y.) 143, 357 (1982).

[27] K. Kamimura, "Singular Lagrangian And Constrained Hamiltonian Systems: Symmetry And Conservation Law," Print-82-0715 (Florence) (unpublished).

[28] X. Gracia and J. M. Pons, Ann. Phys. (N.Y.) 187, 355 (1988).

[29] M. Henneaux, C. Teitelboim, and J. Zanelli, Nucl. Phys. B332, 169 (1990).

[30] J. Gomis, M. Henneaux, and J. M. Pons, Classical Quantum Gravity 7, 1089 (1990).

[31] L. Lusanna, Riv. Nuovo Cimento 14, 1 (1991).

[32] R. Casalbuoni and J. Gomis, (to be published).

[33] R. Casalbuoni, J. Gomis, and G. Longhi, Nuovo Cimento A 24, 249 (1974). 